\title{
Integration of Pharmacogenetics and Pharmacogenomics in Drug Development: Implications for Regulatory and Medical Decision Making in Pediatric Diseases
}

\author{
Chiara Piana, MSc, Linda Surh, MD, PhD, Sabine Furst-Recktenwald, $M D$, \\ Achille Iolascon, $M D, P h D$, Evelyne M. Jacqz-Aigrain, $M D$, $P h D$, Ineke Jonker, $P h D$, \\ Roberta Russo, MSc, Ron H.N. van Schaik, PhD, Judith Wessels, PharmD, PhD, \\ and Oscar E. Della Pasqua, MD, PhD
}

\begin{abstract}
This article aims to provide an overview of the current situation regarding pharmacogenetic and pharmacogenomic $(P G)$ studies in pediatrics, with a special focus on the role of $P G$ data in the regulatory decision-making process. Despite the gap in pharmacogenetic research due to the lack of translational studies in adults and children, several technologies exist in drug development and biomarkers validation, which could supply valuable information concerning labeling and dosing recommendations. If performed under strict good clinical practice quality criteria, such findings could be included in the submission package of new chemical entities and used as additional information for prescribers, supporting further evaluation and understanding of the efficacy and safety profile of new medicines. Even though regulatory authorities may be aware of the potential role of PG in medical
\end{abstract}

practice and guidances are available about the integration of $P G$ in drug development, most data obtained from $P G$ studies are not used by prescribers. The challenge is to better understand whether PG markers can be used to assess potential differences in drug response during the clinical program, so $P G$ data can be integrated into the regulatory decisionmaking process, enabling the introduction of labeling information that promotes optimal dosing in the pediatric population.

Keywords: Pharmacogenetics; regulatory/scientific affairs; clinical pharmacology; pediatric drug development; medical practice

Journal of Clinical Pharmacology, 2012;52:704-716

(c) 2012 The Author(s)
From the Division of Pharmacology, Leiden/Amsterdam Center for Drug Research, Leiden University, the Netherlands (Ms Piana, Dr Della Pasqua); CEDD Global Regulatory Affairs, Neurosciences and Pharmacogenetics, GlaxoSmithKline, Stockley Park, UK (Dr Surh); Translational Medicine, F. Hoffmann-La Roche Ltd., Basel, Switzerland (Dr Furst-Recktenwald); Department of Biochemistry and Medical Biotechnologies, University Federico II of Naples and CEINGE-Advanced Biotechnologies, Naples, Italy (Dr lolascon); Department of Paediatric Pharmacology and Pharmacogenetics, ClC Inserm 9202, Hopital Robert Debré, Paris, France (Dr Jacqz-Aigrain); Xendo Pharma Services B.V. Division Regulatory Affairs, Leiden, the Netherlands (Dr Jonker); CEINGE-Advanced Biotechnologies, Naples, Italy (Dr Russo); Department of Clinical Chemistry, Erasmus MC, Rotterdam, the Netherlands (Drvan Schaik); Department of Clinical Pharmacy and Toxicology, LUMC, Leiden, the Netherlands (Dr Wessels); and Clinical Pharmacology \& Discovery Medicine, GlaxoSmithKline, Stockley Park, UK (Dr Della Pasqua). Submitted for publication March 27, 2009; revised version accepted January 22, 2011 . Address for correspondence: Oscar Della Pasqua, Division of Pharmacology, Leiden/Amsterdam Center for Drug Research, PO Box 9502, 2300 RA Leiden, the Netherlands; e-mail: odp72514@gsk.com. DOI: $10.1177 / 0091270011401619$

\section{INTRODUCTION}

The availability of genetic and genomic data has been considered an important breakthrough in the evaluation of differences in disease susceptibility, progression, and prognosis. Likewise, access to pharmacogenetic information has been deemed important to characterize and explain the variability in treatment response.

Currently, assessment of the clinical relevance of these factors by regulatory agencies relies on voluntary data submission. However, little attention has been paid to 2 important requirements for accurately interpreting such data: (1) validation of the methods and (2) integrated approach, including other relevant covariates. This latter point is critical for pediatric diseases, as true genetic differences must be distinguished from the effects of developmental growth in 
children. The challenge for the coming years will be therefore the development of methodology for the appropriate integration of data on genes, phenotypes, and other biological markers to explain variability in drug response in children, taking into account the role of other important confounders.

Based on this perspective, the integration of novel methodologies in conjunction with advanced statistical models is likely to become critical for the successful evaluation of genetic associations in drug response and subsequent incorporation of clinically relevant findings into the label of medicinal products. Consequently, a key step in the translation of pharmacogenetic data will be the precise and objective definition of response for both efficacy and adverse events (or various clinical phenotypes).

In this section, we provide an overview of the current situation with regard to the value of translational research as the basis for the optimization of pediatric drug development and its implications for labeling. The hurdles and in particular the fragmentation in the efforts in pharmacogenetic research are highlighted. Throughout the article, the term $P G$ will be used to include both pharmacogenetics and pharmacogenomics. A working definition that involves the study of how interacting systems of genes determine drug response will be used in this article to define PG in general. ${ }^{1,2}$

\section{Developmental Growth and Variability in Treatment Response in Children}

PG studies are mostly conducted in adult populations. There are a limited number of clinical studies involving children, and among those very few can be considered translational studies. Thus, there is a lack of pediatric PG data that could be used to support drug labeling. It should be noted, however, that despite this situation, research findings not yet included into product labels are being used in clinical practice (eg, pediatric oncology drugs) in a very empirical manner, a practice that may have safety and efficacy implications for this vulnerable population.

In addition to practical and ethical issues, one of the main challenges regarding the evaluation of PG factors in children is how to account for the confounding effect of developmental growth and variability in individual response. Diseases tend to differ between infants and children, as well as between children and adults, suggesting that relationships between genotype and phenotype may also be different. Therefore, the extrapolation of adult pharmacogenetic data to pediatric patients may not be straightforward depending on the clinical condition and the age bracket being compared. Moreover, metabolic or physiological maturation is the result of complex and continuous processes, from the prenatal period to mid-childhood to adolescence, ${ }^{3}$ such that age-specific changes in pharmacokinetic and pharmacodynamic parameters play a crucial role in pediatric drug response. ${ }^{4,5}$

Biological variability is the second issue to be considered in the evaluation of PG factors in children. Variability to drugs is well documented: among patients treated with standard dosage schedules, $20 \%$ to $30 \%$ will experience poor efficacy, whereas $5 \%$ to $15 \%$ will have safety concerns. ${ }^{6,7}$ Quite often such findings are associated with differences in systemic exposure to a drug (ie, between-subject variability in metabolic clearance) or with disease factors (ie, between-subject variability in drug potency or intrinsic activity).

The evaluation of PG data in drug development is aimed at identifying the sources of interindividual variability in drug- and disease-related factors, so that one can ultimately explain differences in drug response across the population. In fact, on the basis of these premises, pharmaceutical companies have systematically avoided the development of compounds that show elimination via polymorphic metabolic routes, such as CYP3A4 substrates. ${ }^{8}$

However, many covariates contribute to variation in response to drugs and thus to between-subject differences. Major covariates typically identified in drug development include adherence (ie, taking a medicine), formulation (ie, differences in bioavailability), and demographic variables (ie, age, gender, lifestyle, etc). Until recently, these intrinsic and extrinsic factors were considered among the main explanatory causes of variability. In contrast, it was less likely for genetic variation or polymorphisms to be considered contributing factors to variation in drug response. This situation is worse in pediatric research, as even less attention is given to developmental pharmacogenomics. For example, some developmental genes, such as those for fetal hemoglobin, are expressed early in life and then are switched off in adulthood. Such gene switching could be associated with differences in the therapeutic effects of drugs, either efficacy or toxicity, but very limited data are available to characterize its clinical relevance. ${ }^{9}$

Despite the clear role of developmental growth on gene expression and subsequent phenotypical differences, drug response in children has much in common with the responses in adults and other mammals. ${ }^{10} \mathrm{In}$ fact, there are cases in which a pediatric indication may be based on controlled and well-established trials in adults, with other information supporting pediatric use, as stated in the Paediatric Rule of 1994. Various 
examples of polymorphisms in pharmacokinetics, pharmacodynamics, and disease can be mentioned, but such a variation does not translate into differences in response between children and adults (eg, antiretrovirals,${ }^{11}$ deferiprone ${ }^{12}$ ). On the other hand, there are genetic variations that lead to differences in response in children and adults, such as methotrexate ${ }^{13}$ antidepressants, ${ }^{14}$ and a few antitumor agents used in neuroblastoma. ${ }^{15}$ The key question remains, therefore, how to discriminate which polymorphisms will show marked differences across populations and, most important, how to assess the clinical relevance of such differences. Such an evaluation demands translational research efforts, which do not only ensure data generation in a systematic manner but also support the use of the model-based approaches for accurate interpretation and extrapolation of findings. ${ }^{16}$

Based on the aforementioned, it becomes evident that the most conservative position is assumed by regulatory authorities when data are lacking. As a consequence, drug effects in adults are considered to differ from children until proven otherwise. This perception often arises because of historical reasons (ie, most drugs have not been adequately studied in the pediatric population). ${ }^{17}$

\section{Impact of PG on Drug Development and Regulatory Decision-Making Process in Pediatrics}

Three factors need to be taken into account to define efficacy and safety of a drug: drug exposure in relevant tissues (described by the ADME process), the pharmacodynamics of the drug (relationship between drug concentration and effect), and the underlying disease. To date, numerous ADME response-gene data sets ${ }^{18-20}$ are available in adults, which allow understanding of the role of genetic variation in drug-metabolizing enzymes. Considering the impact of PG data in clinical practice, investigations related to drug metabolism have been well accepted in adults but not in children. Generally, ADME data are collected and evaluated in preclinical and early clinical drug development. However, as data mount from the later phases of drug development, this information needs to be put into context with regard to its relevance for treatment response. Without assessing such a relationship, any observed PG differences become ambiguous, making the judgment of such differences rather difficult, if not impossible. An example of such a translational effort is the research on the detailed understanding of the mechanism of action and metabolism of anticoagulants, particularly warfarin, which has led to changes in the drug label by the US Food and Drug Administration, including recommendations related to PG data. ${ }^{21}$ Likewise, the application of PG to pharmacodynamics and ADME properties should not be different in pediatric drug development. ${ }^{22,23}$ However, with the exception of a few examples, the integration of PG has not been performed systematically because of ethical and practical issues in pediatric studies.

The ultimate goal in drug development, irrespective of whether treatment refers to adult or pediatric indications, is not only to deliver drugs of value for patients but also to improve medical practice, such as prescription guidelines and dosing recommendations that are tailored to the specific needs of a patient group or population. In the case of pediatric populations, it is essential to consider how PG data can be effectively used for such purposes. Thus far, this has not really happened in a systematic manner. For regulatory and labeling purposes, one must consider the predictive rather than (simply) prognostic value of PG data, which require the integration of biomarkers of treatment response to pharmacogenetic studies. In fact, a recent mechanistic classification of biomarkers has been proposed, which elegantly establishes the correlation between PG factors to other indicators of physiological function, pathogenic processes, and pharmacological response to a drug. ${ }^{24,25}$ This classification is applicable to any patient population and offers additional advantages for the assessment of specificity and sensitivity of the end points of interest, which are an important component of the validation procedures for any methodology. ${ }^{26,27}$ Unfortunately, validation procedures are lacking for PG data, particularly for data obtained in pediatric disease conditions. In addition, there is another aspect related to validation of biomarkers that needs to be carefully considerednamely, whether it is meant to be used as a decisionmaking tool in drug development or to support medical decisions in clinical practice. In drug development, the objective is to characterize the efficacy and/or safety of drugs as assessed during well-controlled clinical trials. In contrast, in medical practice, the aim is not only to obtain proxys for treatment outcome in terms of safety and efficacy. Rather, the main objective is to facilitate decision making in those conditions for which the absence of such data could have clinical implications, including different diagnosis and changes in the therapeutic management.

These goals are unlikely to be achieved under the current paradigm, which is primarily focused on methods and other technical aspects of data acquisition. The gap and challenges for data integration and interpretation have been taken for granted and left out of the scope of most regulatory and scientific initiatives. 


\section{PG IN PEDIATRIC DRUG DEVELOPMENT}

\section{Current Approaches in PG Research}

In a previous publication by our group, we have highlighted the lack of a well-defined, hypothesis-driven strategy for translational PG research, which has prevented the interpretation of findings for regulatory purposes and subsequently incorporation of the PG information into the label. ${ }^{28}$ Despite the aforementioned validation issues, claims continue to be made about PG data, without taking into account the variety of methodologies from which they arise. In fact, the need for standardized techniques in PG research protocols has not been perceived yet, nor is its potential impact on the use of PG data in the regulatory processes.

Current research protocols can be classified in 2 categories: the first approach is based on association studies of drug response with single-nucleotide polymorphisms (SNPs) in "candidate genes," which are genes selected according to their putative role in pharmacokinetics and pharmacodynamics using a hypothesis-driven approach. The second approach intends to associate independently evenly distributed SNPs across the human genome in (or in linkage with) candidate genes with response, without using any a priori knowledge about the pharmacology of a drug, that is, a hypothesis-free approach.

Although the candidate gene approach is appealing, it may fail for several reasons. First, most methods do not take into account the potential role of other genes, including those genes whose function is not yet understood. In addition, it does not account for gene duplication and other mechanisms that alter protein expression, such as gene-environment interactions. Also the genome-wide analysis (GWA) of SNPs has important limitations, especially with regard to the inferences that can be made from such findings. Among other issues, confounders are often present, which lead to the identification of noncausal correlations between gene and phenotype. In addition, sophisticated statistical approaches may be required to account for multiplicity of testing. These limitations are further compounded by the difficulties in assessing clinical relevance, as the studies from which these data arise were not designed for the assessment of PG as primary endpoints. An interesting step between the candidate gene and the whole-genome PG analysis is the study of drug response in downstream and interacting signaling pathways. Pathways of genes with allelic variants may be more important than individual genes, with the effects of polymorphisms in networks of genes acting together to create one phenotype. In addition, it has to be realized that genetic factors are estimated to account for $15 \%$ to $30 \%$ of the interindividual variation in drug response. Thus far, only for certain drugs an exceptionally high degree of interindividual variation in treatment response has been observed, and such findings were usually attributed to genetic variants in pharmacodynamics or disease. For that reason, drug efficacy and safety are likely to be a complex result of environmental (extrinsic) and endogenous (intrinsic) factors with the influence of many interacting genes. Moreover, it should be noted that drug treatment itself may modulate or alter phenotypical features. Therefore, translation of pharmacogenetic data into label claims or dosing recommendations may require both prospective and retrospective evaluation of candidate genes in well-defined case control cohorts. Such a learning-confirming paradigm is essential to ensure accurate assessment and interpretation of the clinical relevance of PG data.

From the data analysis perspective, readers should be aware that use of naive pooling and other nonparametrical approaches predominates in PG research. These methods do not account for the variance structure and often do not consider longitudinal factors, as the assumption is that genotype does not change over time. This assumption ignores the fact that gene expression and other phenotypical data are often more informative or correlate better with variability in disease and drug effects. Multivariate and hierarchical models are urgently needed for the accurate evaluation of drug-gene associations. For instance, research on the role of PG differences in drug metabolism often neglects variation due to the effect of formulation, which may be observed as within-subject or between-subject variability depending on the study design. Likewise, poor adherence to treatment is well known to be the ultimate cause of lack of response or increase in adverse events. PG research protocols ignore the impact of patient behavior with regard to adherence, which has been shown not to be random across treatment arms and dose levels.

Therefore, statistical methods are needed that ensure common factors in variation to drug response are first addressed before polymorphisms are evaluated to identify the correct source and explain variation in response. This implies the use of parametric approaches in which genetic and nongenetic factors-important for the evaluation of phenotype associations-are integrated and analyzed concomitantly. The use of Bayesian hierarchical models and nonlinear mixed effects modeling (population approach) may be a powerful tool to investigate the relationships between genetic and nongenetic covariates and pharmacokinetic/ pharmacodynamic parameters. In conjunction with 
clinical trial simulation techniques, such models make it possible to explore the implications of different clinically relevant scenarios and describe variability in drug response across specific groups of the population.

Another important problem is that scientific journals and research groups tend to only publish positive association results while potentially neglecting important study quality issues such as the power and sample size of the study needed to detect so-called true associations in complex phenotypes. Preferentially, both negative and positive associations with polymorphisms and drug response should be reported to make meta-analysis of data possible. As already mentioned earlier, the challenge has become the development of methodology for appropriate integration of data on genes, phenotypes, and other biological markers to explain variability in drug response in children, taking into account the confounders of study design. Epidemiologic research, gene expression analyses, and proteomic methods (in target tissues) with sophisticated statistical models are likely to be important for the successful evaluation of genetic associations in drug response. Several suggestions can be made for conducting studies to develop polygenetic models for drug response. However, the key step in any clinical PG study is the precise and objective definition of response for both efficacy and adverse events (or various clinical phenotypes). If efficacy and safety outcomes are not clearly defined, PG variables related to the outcome become useless.

It should be clear that most treatment-gene associations of clinical relevance and higher frequency will be of small effect and multifactorial. In contrast, many of the PG polymorphisms in drug disposition that have been well studied and published (eg, cytochrome P450) tend to be of large effect, are rare depending on the ethnic background, and involve a single gene (or a few genes). Nevertheless, neglecting the impact of nongenetic factors on drug response will lead to confounded associations whether multifactorial or single-gene drug response is involved. Therefore, to account for the "noise" in the data, one must bear in mind issues such as statistical power and stratification, ensuring uniform criteria for patient inclusion and exclusion. Moreover, protocol design should consider the assessment of other disease characteristics, as well as demographic and environmental factors as covariates for response, and incorporate differences in drug exposure and measures of treatment compliance. In addition to the validation and data analysis aspects, prospective multicenter PG studies must comply with the regulations regarding the handling of PG samples and relevant analytical procedures, as defined by International Conference on Harmonisation (ICH) E6 guidelines. ${ }^{29}$
Unfortunately, even under these circumstances, protocol designs may not be suitable for establishing associations between treatment response and PG, particularly if the primary objective of the protocol is efficacy (ie, active vs control) and subjects have not been stratified accordingly across treatment arms. Given the dynamic, reversible nature of some efficacy and toxicity endpoints, associations may be missed if exposure-response relationships are not known.

\section{Validation of Pharmacogenetic Biomarkers}

As already outlined, the accurate use of PG markers for decision making demands further efforts on validation. The validation requirements will vary depending on the intended application or stakeholder, such as researcher, regulator, medical practitioner, or patient. Appropriate study size is of great importance, as well as the confirmation of findings in independent patient groups. As a matter of fact, case control cohorts have been proposed and performed in a number of cases. For instance, the effect of the polymorphism in the $A B C B 1$ gene in antidepressant therapy has been shown for those drugs that have been identified as substrates of $A B C B 1 .{ }^{30}$ However, even in the case of case-control cohorts, PG markers may not be valid for all populations, either because of differences in genetic background or because of environmental factors. This implies that validation of PG markers across different groups is important and necessary. In this respect, when a genetic polymorphism has been demonstrated to mechanistically affect a specific drug pathway, the chances of finding this marker to be valid across patient groups is substantially larger, which justifies that research should be done to find the explanation for an observed PG association.

On another aspect, one should realize that age can have a profound effect on the validity of PG markers. For example, because of the maturation processes of drug-metabolizing enzymes, genetic polymorphisms in the genes encoding these enzymes will not have the same impact in adults and children, particularly neonates and young infants. ${ }^{31}$ Therefore, PG markers that are to be used in these populations require validation with studies involving the appropriate age range. Subsequently, such markers may be incorporated into patient diagnostic procedures only after they have been validated in the appropriate patient population and their effect has been identified mechanistically and proven clinically useful in prospective randomized trials.

The influence of environment and clinical history on a patient's phenotype also needs to be taken into account because the phenotype could interact with 
the genotype and consequently affect the clinical response of the patient. In addition, during biomarker validation, procedures and quality control measures should be in place to identify sampling errors, so that methodological limitations can be clearly discriminated from procedural issues. Of particular interest are the estimates of sensitivity and specificity for a given method because important decisions on drug prescription may follow from the results of such a test or diagnostics. For example, the implementation of 2 different genotyping platforms for identifying the same SNP should be considered. In the experience of one of the authors, it was observed that some genotyping techniques may misclassify $\geq 1 \%$ of the samples (unpublished data). Of course, the importance of this bias will depend on the frequency of the polymorphism and/or if adverse events are associated with it. Typically, the implications of such a misclassification may be minimized quickly if other phenotype information or therapeutic drug monitoring is available. However, under these circumstances, one may question the clinical value of such a PG marker.

The impact of the lack in assay sensitivity and/or assay specificity needs therefore to be carefully evaluated in light of the overall benefit-risk ratio. First, it is important to establish if PG-specific data need to be integrated with any other known covariates for a given drug or disease condition. When treating patients, it is the clinical sensitivity/specificity that is required for decision making, not the sensitivity/specificity of the PG technique or method itself. However, it is also true that clinical decisions often have to be made based on incomplete information regarding each individual covariate or its interactions. Therefore, it is important to distinguish clinical validation from assay validation. In fact, many readers may be unaware that in comparison to new DNA methodologies, many clinical chemistry tests typically have far lower assay specificity and/or sensitivity. ${ }^{32}$ This limitation has been often overlooked in most published literature regarding PG research. From a therapeutic perspective, it is essential to make a comprehensive assessment of the impact of false negatives and false positives arising from technical or methodological procedures as they may determine the value of such markers for clinical decision making. An immediate consequence of the differences in specificity and sensitivity is that accurate interpretation of PG differences may be limited to the population level but not necessarily valid for individual patients.

The integration of PG data into regulatory submissions will have to take into account the aforementioned limitations. A case-by-case approach may not be sufficient to warrant effective use of the PG data because of the exploratory nature of research protocols and empiricism in evidence-synthesis in medical practice. Thus, guidelines will need to focus on scientific principles that stand the test of time and incorporate flexibility as both technology and medical understanding evolve. Model-based statistical methods must be considered to translate the evidence from PG differences into specific dose recommendations and/or actions for prescriber and patient.

\section{REGULATORY CONSIDERATIONS ON THE USE OF PG DATA TO ENHANCE THE DEVELOPMENT OF NEW MEDICINES}

\section{PG and the Regulatory Agencies}

There is wide recognition by regulatory authorities that biomarkers, especially PG, have the potential to improve the discovery, development, and use of medicines. ${ }^{33}$ The Food and Drug Administration (FDA) has indicated in its Critical Path Initiative that a new generation of predictive biomarkers (ie, PG biomarkers) is one of the most important areas for improving medical product development. ${ }^{34,35}$ The European Medicines Agency (EMA) also mentions PG as a challenging new technology in its Road Map to $2010 .^{36}$ Overall, individual regulatory agencies have issued various white papers, points to consider, draft guidances, and final guidances to integrate PG into drug development. As such, regulatory guidance documents become important in steering operating practices for sponsors of pharmaceutical research and development. ${ }^{37}$

In addition to such guidances, a few other initiatives reflect the need for creating a stronger scientific basis for decision making in regulatory processes. For instance, the regulatory agencies in the United States, Europe, and Japan have introduced new practices and hired additional personnel to facilitate early contact with sponsors, encouraging among other things the submission of PG data. These changes derive from an initial concept of "safe harbor," which has progressed, particularly in the United States, to the voluntary genomic data submission (VGDS) and, more recently, to the acceptance and inclusion of other exploratory biomarkers (voluntary exploratory data submission [VXDS]).

However, although the focus of regulators on safety and efficacy involves consideration of predefined criteria for the review of clinical trial submissions, VGDS has been used to promote emerging biomarkers specific to drug development, without considering (1) the validation of the methods and (2) the integration of other relevant covariates. In this respect, it is surprising that 
pharmacokinetic/pharmacodynamic (PK/PD) modeling has not been considered yet as the approach of choice for covariates analysis and ultimately to assess the relevance of PG differences together with other influential factors. In conjunction with clinical trial simulations, it is possible to quantify the interaction between all relevant factors explaining between-subject differences and thereby assess the contribution and clinical implications of PG, as compared with the overall variability.

Recent agreements among the 3 major geographic regions have also led to combined efforts in regulatory guidelines as represented by the ICH on the following: (a) ICHE15 guidance on "Definitions for Genomic Biomarkers, Pharmacogenomics, Pharmacogenetics, Genomic Data and Sample Coding Categories" and (b) the recently proposed ICH E16 draft guidance on "Pharmacogenomic Biomarker Qualification: Format and Data Standards." Thus, PG has become a key scientific topic to which regulatory agencies are proactively engaged in evidentiary progress to understand the value of PG data for future regulatory applications such as the assessment of dose selection, safety, and efficacy. ${ }^{33,38}$ It is unfortunate that no actions have been taken yet to endorse the importance of model-based data analysis methodologies.

Ultimately and ideally, the path in drug development culminates with the regulatory approval of a new chemical entity. But it does not end there. A major part of the regulatory approval process is the identification of relevant data from across extensive evidence accumulated over a long time span to justify and support the claims in the label of a new medicine. Just as with other types of data sets, the integration of PG into a label must fulfill similar stringent clinical science criteria and meet prescriber as well as patient needs. Thus, these needs are not only to effectively and safely use a medicinal product but also to understand its benefits and risks, as is practicably feasible with the available data at the time of approval. It should also be noted that regulatory approvals are no longer considered endpoints but are now an en route milestone, as the data collection continues postmarketing for pharmacovigilance and further understanding of the overall clinical utility. As already explained, assessment of the contribution of PG differences to changes in the risk-benefit ratio and consequently its implications for patients and prescribers are likely to remain wishful thinking without guidelines aimed at (1) the requirements for integration of PG data and (2) the methodology for the analysis of the results in conjunction with other relevant factors contributing to between-subject variability in treatment response.

\section{Applications of PG in Drug Development and on the Medicine Label}

It is important to differentiate the use of PG in drug development from PG on a medicine label or PG within medical practice or PG in peer-reviewed research. There are only limited PG assays or diagnostics available for human application in general. PG diagnostics are primarily related to drug metabolism because this is the main area in which there is mechanistic evidence and some understanding of the clinical usefulness of the data. Some examples associated with patient screening procedures or dosing algorithms are found in early phase drug development or when an adverse event arises for a drug that has a narrow therapeutic range. In contrast, the objective of a label is primarily to guide the prescriber for the safe and efficacious use of a medicine. To this purpose, quantitative methods are required that take into account biological differences, possible dosing regimens, and the decision criteria and course of actions to be taken by the prescriber.

Such an approach differs considerably from the policy currently implemented by some regulatory agencies. The FDA has published a comprehensive list on the use of PG data and established a definition of "valid" genomic biomarkers in the context of FDAapproved medicine labels. ${ }^{39}$ About $10 \%$ of these labels contain some PG information, but this number appears to be slowly increasing during the past decade. In the last update from the first quarter of 2008, a PG test was "required" or "recommended" for 10 of the 17 different biomarkers mentioned in the labels. Nevertheless, the differences between the advancement of these concepts in adult as compared to pediatric indications become evident from the fact that among these recent 10 biomarkers, only very few are approved for pediatric use: CCR5 chemokine C-C motif receptor as a required test in the label for maraviroc (from 16 years on), G6PD deficiency as a recommended test in the label for rasburicase (from 1 month on) or dapsone (from 12 years on), test for thiopurine methyltransferase (TPMT) variants recommended in the thioguanine and mercaptopurine label (no age limitations), and the urea cycle disorder deficiency test for valproic acid (over 2 years).

In summary, it generally appears that most PG information is descriptive with no specific action for the prescriber or patient (such as diagnostic testing or change of dosing or choice of drug)—all of which reflects the lack of PG data and of quantitative methods for the assessment of its clinical relevance, including subsequent prescriber actions. Of note and just as important, it should be clear that the value of additional PG data remains unknown or hypothetical until sufficient 
evidence demonstrates its relevance. What is certainly critical in this process is the need to assess the clinical utility, which can only be accurately evaluated if PG data are analyzed in an integrated manner with other pharmacokinetic, pharmacodynamic, and disease variables. Thus, it is not surprising that only a few labels have recommended actions (typically for safety issues) and even fewer require or mandate a diagnostic PG test before being able to prescribe the specific medicine.

Depending on the clinical use of PG data in drug development, there are different levels of validation requirements in PG for assay versus medical interpretations. Regarding laboratory techniques or test procedures, validation is a common practice both for (bio) analytical methods used in drug development and for in vitro diagnostics ${ }^{40}$ in which the focus is on assay characteristics (accuracy, sensitivity, specificity). These principles can be applied to the methodologies used to generate PG data. In comparison, for regulatory purposes, validation should be consistent with approaches applied to other types of data sets used in drug development. Thus, early stage protocols should be exploratory and aimed at finding relevant clinical associations with a biomarker, whereas later stage protocols should yield confirmatory evidence of the correlation between PG differences and a clinical endpoint (efficacy or safety). Therefore, the validation of PG biomarkers for clinical use requires a different type of data set than assay validation and needs to be fit for purpose. These differences imply that PG data generation may demand specific protocols, which may have ethical and practical implications if implemented during drug development.

A clear distinction is therefore needed with regard to the requirements for the generation of PG data before and after market approval of a new medicine. Given that the volume of PG data emerging from human protocols is increasing, there will be differing opinions about its relevance until evidence for treatment-gene interactions is established with some confidence. However, one of the major advantages of PG in drug development is the potential to explain or predict some of the variability in drug response. Such information can be used in protocol design so as to screen, enrich, and/ or guide dose titration during clinical evaluation of new medicines. In contrast, to define PG-related recommendations in medical practice, extensive genetic epidemiology data spanning over many patient-years are required. Such a volume of data cannot be easily obtained during drug development, particularly when the prevalence of the genetic variants is low. In any case, accurate clinical validation requires the use of $\mathrm{PK} / \mathrm{PD}$ modeling and simulation, as it ensures integration of PG data to other influential covariates and enables optimization of prospective study protocols.

Even if associations are established demonstrating the clinical relevance of PG differences, increased complexity of treatment needs to be taken into account, and as for other types of medical data, such criteria should only be applied when there is significant improvement of the benefit/risk for the patient. Also, cultural and education hurdles exist that need to be overcome if PG is to become part of the standard toolkit in therapeutics. Physicians are not trained to dose titrate or classify patients based in terms of their genotype. The same type of training is required in clinical pharmacology to ensure proper interpretation of PG data and subsequent translation of findings into labeling recommendations. Certainly, ancillary technologies are required for medical and patient education before PG can become a reality in everyday life therapeutics. Identification of a genotype does not solve the problem of optimal dosing or treatment prognosis. Hardly feasible at present, dosing algorithms need to evolve in parallel to pharmaceutical formulation technologies to enable dose titration, fractioning, or tailoring while minimizing the risk of medical prescription errors, which are critical in adults but even more so in pediatric patients. Thus, there are practical challenges for the integration of PG into medical practice, which demand concerted efforts by industry, regulatory agencies, and health care professionals.

\section{OVERVIEW OF PG DATA IN PEDIATRIC DISEASES SUMMARIZED PER THERAPEUTIC AREA}

To gather evidence of the challenges and issues described in the previous sections, a literature review was performed to summarize some of the existing literature data regarding PG studies in the pediatric population. Data have been classified into different therapeutic areas to facilitate further understanding of disease-related factors and possibly fill the current gaps in PG knowledge in young patients.

From Table I, it is clear that the clinical relevance of PG information and subsequent impact on prescription are variable. Even in those cases where changes in prescription practice are recommended, there is little research on the benefit of screening, taking into account allele and disease prevalence as well as specificity and sensitivity of the methods. An analysis of health claims data suggests that avoiding the burden of ineffective treatment is likely to offset the costs of screening the population of primary care patients with asthma-like symptoms for treatment response, ${ }^{41}$ but 


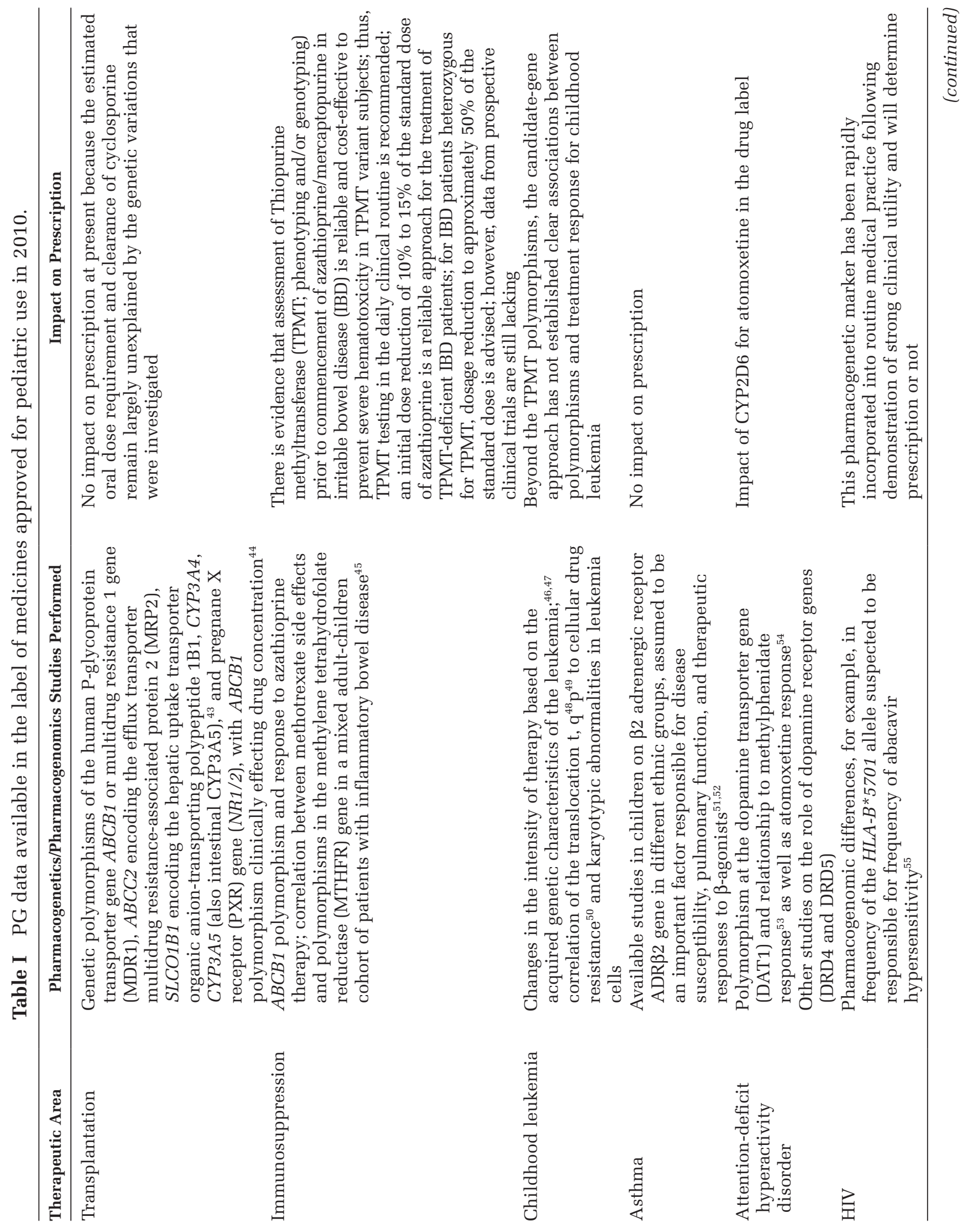




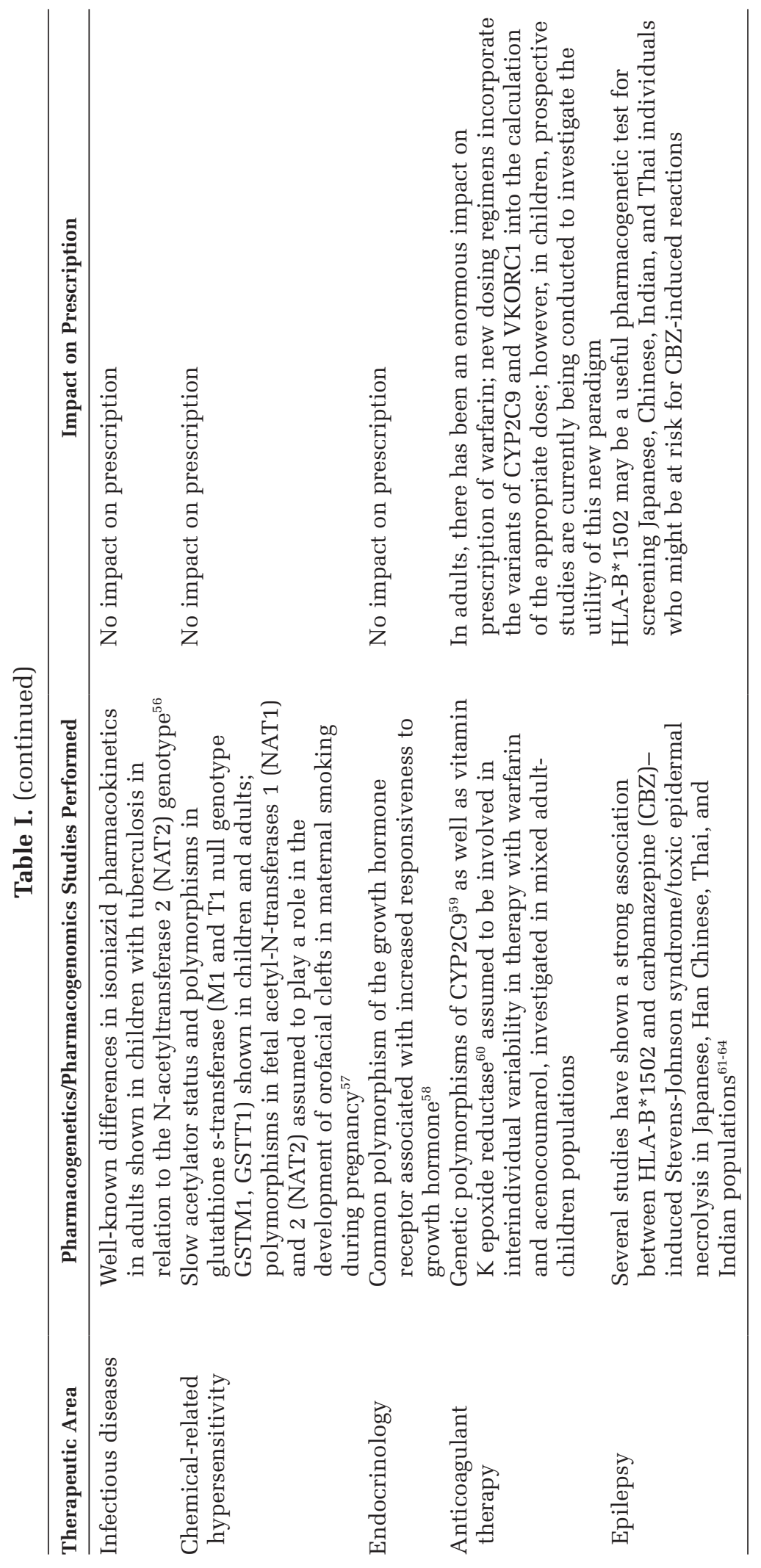


thus far, similar analyses have not been performed for other diseases.

Irrespective of whether PG or other biomarkers refer to pediatric or adult diseases, the challenge is collecting the relevant data based on a precise and objective definition of response for both efficacy and adverse events. Subsequently, once appropriate data have been collected, 2 issues should be considered in the analysis and interpretation of the available information. First, a quantitative, model-based evaluation should be performed of the PG differences. ${ }^{16}$ The second issue pertains to the need for updated PG information, which reflects changes in clinical relevance. In this respect, the contribution of collaborative networks aimed at identifying genomic markers in the pediatric population and subsequent data sharing is becoming increasingly important. ${ }^{42}$

\section{CONCLUSIONS}

Within drug development, PG is high on the agenda of regulatory authorities worldwide. There is now broad awareness on the potential of PG biomarkers and their contribution to the scientific evidence in support of drug approval. However, most PG may not qualify from a regulatory perspective toward actionable decisions by prescribers to alter key medical outcomes and thus be included on a medicine label. Yet, within drug development, there are key steps to which PG could be integrated to assist the progression of a new chemical entity more efficiently or to terminate faster. Even though the details of the actual PG assay or test do not end up on the label or in medical practice, such PG data may contribute to decision making and go/no-go criteria used prior to the approval of the medicinal product. In medical practice for both adult and pediatric patients, the potential relevance of PG for personalized medicines is as yet limited and should only be applied when clear benefit for the patient is justified in a transparent, scientific, and systematic way.

It is surprising that none of the initiatives undertaken to characterize the role of PG factors has considered the need to integrate other clinical, pharmacological, and demographic factors. Although modeling and simulation (M\&S) have gained relevance as a quantitative, integrative tool in drug development and therapeutics, it is wearisome to see the role of PG disconnected from all other factors (covariates) contributing to clinical outcome. Moreover, it should be noted that because of the interaction between various drug- and disease-related factors, many of the associations may not be identified, even if statistical power is formally taken into account.
Given that genetic factors must be considered within a specific clinical context, and in conjunction with environmental factors, different scenarios are required to assess the added value of PG both in drug development and/or in medical practice. Obviously, this is a complex question that involves not only the cost of the PG test itself but also other feasibility issues. In pediatric diseases, these considerations include long-term effects.

Drug development and drug therapy in children have to deal with practical and ethical hurdles, which increase the challenges for the effective collection, use, analysis, and interpretation of PG data. Yet, even with these barriers, the availability of PG biomarkers may make a difference, ensuring that children receive the dose and/or drug that is the most effective and least harmful for them.

\section{BULLET POINTS}

- Currently, assessment of the clinical relevance of these factors by regulatory agencies relies on voluntary data submission. However, little attention has been paid to 2 important requirements for accurately interpreting such data: (1) validation of the methods and (2) integrated approach, including other relevant covariates. This latter point is critical for pediatric diseases, as true genetic differences must be distinguished from the effects of developmental growth in children.

- The challenge for the coming years will be the development of methodology for appropriate integration of data on genes, phenotypes, and other biological markers to explain variability in drug response in children. Consequently, a key step in the translation of pharmacogenetic data will be the precise and objective definition of response for both efficacy and adverse events (or various clinical phenotypes).

- Because important decisions on drug prescription may follow from the results of tests or diagnostics, modelbased approaches (M\&S) are recommended as a tool for clinical validation. They ensure integration of PG data with other influential covariates, providing quantitative estimates of the risk-benefit ratio for individual patients across a range of clinically relevant scenarios.

- A clear distinction is needed with regard to the requirements for the generation of PG data before and after market approval of a new medicine. One of the major advantages of PG in drug development is the potential to explain or predict some of the variability in drug response. Such information can be used in protocol design so as to screen, enrich, and/or guide dose titration during clinical evaluation of new medicines.

- In contrast, to define PG-related recommendations in medical practice, extensive genetic epidemiology data spanning over many patient-years are required. Such 
a volume of data cannot be obtained during drug development, particularly when the prevalence of the genetic variants is low.

Financial disclosure: This publication is the result of concerted efforts by the TEDDY Network of Excellence, a project under the auspices of the European Union's Sixth Framework Programme.

\section{REFERENCES}

1. Klein TE, Chang JT, Cho MK, et al. Integrating genotype and phenotype information: an overview of the PharmGKB project. Pharmacogenetics Research Network and Knowledge Base. Pharmacogenomics J. 2001;1:167-170.

2. Leeder JS. Developmental and pediatric pharmacogenomics. Pharmacogenomics. 2003;4:331-341.

3. Leeder JS. Translating pharmacogenetics and pharmacogenomics into drug development for clinical pediatrics and beyond. Drug Discov Today. 2004;9:567-573.

4. Bartelink IH, Rademaker CM, Schobben AF, van den Anker JN. Guidelines on paediatric dosing on the basis of developmental physiology and pharmacokinetic considerations. Clin Pharmacokinet. 2006;45:1077-1097.

5. Kearns GL, Abdel-Rahman SM, Alander SW, Blowey DL, Leeder JS, Kauffman RE. Developmental pharmacology—drug disposition, action, and therapy in infants and children. $N$ Engl J Med. 2003;349:1157-1167.

6. Zhou SF, Di YM, Chan E, et al. Clinical pharmacogenetics and potential application in personalized medicine. Curr Drug Metab. 2008;9:738-784

7. Roden DM, George AL Jr. The genetic basis of variability in drug responses. Nat Rev Drug Discov. 2002;1:37-44.

8. Kapelyukh Y, Paine MJ, Marechal JD, Sutcliffe MJ, Wolf CR, Roberts GC. Multiple substrate binding by cytochrome P450 3A4: estimation of the number of bound substrate molecules. Drug Metab Dispos. 2008;36:2136-2144.

9. Pearce S, Budge H, Mostyn A, et al. Prolactin, the prolactin receptor and uncoupling protein abundance and function in adipose tissue during development in young sheep. J Endocrinol. 2005;184:351-359.

10. Cappon GD, Bailey GP, Buschmann J, et al. Juvenile animal toxicity study designs to support pediatric drug development. Birth Defects Res B Dev Reprod Toxicol. 2009;86:463-469.

11. Tozzi V. Pharmacogenetics of antiretrovirals. Antiviral Res. 2010;85:190-200.

12. Limenta LM, Jirasomprasert $T$, Tankanitlert J, et al. UGT1A6 genotype-related pharmacokinetics of deferiprone (L1) in healthy volunteers. Br J Clin Pharmacol. 2008;65:908-916.

13. Becker ML, Leeder JS. Developmental pharmacogenetics in pediatric rheumatology: utilizing a new paradigm to effectively treat patients with juvenile idiopathic arthritis with methotrexate. Hum Genomics Proteomics. 2010;2010:257120.

14. Sjoqvist F, Bertilsson L. Clinical pharmacology of antidepressant drugs: pharmacogenetics. Adv Biochem Psychopharmacol. 1984;39: 359-372.
15. Bellanti F, Kågedal B, Della Pasqua O. Do pharmacokinetic polymorphisms explain treatment failure in high-risk patients with neuroblastoma? Eur J Clin Pharmacol. 2011 Feb 2. [Epub ahead of print] 16. Bellanti F, Della Pasqua O. Modelling and simulation as a research tool in paediatric drug development. Eur J Clin Pharmacol. 2011 Jan 19. [Epub ahead of print]

17. Stephenson T. How children's responses to drugs differ from adults. Br J Clin Pharmacol. 2005;59:670-673.

18. Ekins S. Systems-ADME/Tox: resources and network approaches. J Pharmacol Toxicol Methods. 2006;53:38-66.

19. Koshy B, Miyashita A, St Jean P, et al. Genetic deficiency of plasma lipoprotein-associated phospholipase A2 (PLA2G7 V297F null mutation) and risk of Alzheimer's disease in Japan. J Alzheimers Dis. 2010;21:775-780.

20. Sofat R, Casas JP, Kumari M, et al. Genetic variation in complement factor $\mathrm{H}$ and risk of coronary heart disease: eight new studies and a meta-analysis of around 48,000 individuals. Atherosclerosis. 2010;213:184-190.

21. Hill CE, Duncan A. Overview of pharmacogenetics in anticoagulation therapy. Clin Lab Med. 2008;28:513-524.

22. Gaedigk A, Eklund JD, Pearce RE, et al. Identification and characterization of cytochrome P4502D6*56B (CYP2D6*56B), a novel allele associated with the poor metabolizer phenotype. Clin Pharmacol Ther. 2007;81:S85.

23. Allegaert K, Verbesselt R, Naulaers G, et al. Developmental pharmacology: neonates are not just small adults. Acta Clinica Belgica. 2008;63:16-24.

24. NIH Biomarkers Definitions Working Group. Biomarkers and surrogate endpoints: preferred definitions and conceptual framework. Clin Pharmacol Ther. 2001;69: 89- 95.

25. Danhof M, Alvan G, Dahl SG, Kuhlmann J, Paintaud G. Mechanism-based pharmacokinetic-pharmacodynamic modeling: a new classification of biomarkers. Pharm Res. 2005;22:1432-1437.

26. Vickers AJ, Wolters T, Savage CJ, et al. Prostate specific antigen velocity does not aid prostate cancer detection in men with prior negative biopsy. J Urol. 2010;184:907-912.

27. Shariat SF, Lotan Y, Vickers A, et al. Statistical consideration for clinical biomarker research in bladder cancer. Urol Oncol. 2010;28:389-400.

28. Krekels EHJ, van den Anker JN, Baiardi P, et al. Pharmacogenetics and paediatric drug development: issues and consequences to labelling and dosing recommendations. Expert Opin Pharmacother. 2007;8:1787-1799.

29. International Conference on Harmonisation (ICH). ICH Topic E 6 (R1) Guideline for Good Clinical Practice. Geneva, Switzerland: ICH; 2010.

30. Uhr M. ABCB1 genotyping is crucial for treatment with drugs that are P-glycoprotein substrates. Biol Psychiatry. 2005;57:78S.

31. de Wildt SN, Kearns GL, Hop WCJ, Murry DJ, Abdel-Rahman SM, van den Anker JN. Pharmacokinetics and metabolism of intravenous midazolam in preterm infants. Clin Pharmacol Ther. 2001; 70:525-531.

32. Surh LC, Lesko LJ, Hobbs S, et al. Fit-for-purpose pharmacogenomic biomarkers in drug development: a project team case study with 'what-ifs'. Pharmacogenomics. 2009;10:137-147.

33. International Conference on Harmonisation (ICH). ICH Topic E15-Note for Guidance on Definitions for Genomic Biomarkers, 
Pharmacogenomics, Pharmacogenetics, Genomic Data and Sample Coding Categories (EMEA/CHMP/ICH/437986/2006). Geneva, Switzerland: ICH; 2008.

34. Food and Drug Administration (FDA). Challenge and Opportunity on the Critical Path to New Medical Products. Rockville, MD: FDA; 2004.

35. Food and Drug Administration (FDA). Critical Path Opportunities Report. Rockville, MD: FDA; 2006.

36. European Medicines Agency (EMEA). Road Map to 2010. London: EMEA; 2008.

37. EMEA Work plan for the pharmacogenomics working party. London: EMEA; 2008.

38. Food and Drug Administration (FDA). FDA Guidance for Industry-Pharmacogenomic Data Submissions. Rockville, MD: FDA; 2005.

39. Food and Drug Administration (FDA). Table of Valid Genomic Biomarkers in the Context of Approved Drug Labels. Rockville, MD: FDA; 2008.

40. Stallings SC, Huse D, Finkelstein SN, et al. A framework to evaluate the economic impact of pharmacogenomics. Pharmacogenomics. 2006;7:853-862.

41. Ross CJD, Carleton B, Warn DG, Stenton SB, Rassekh SR, Hayden MR. Genotypic approaches to therapy in children: a national active surveillance network (GATC) to study the pharmacogenomics of severe adverse drug reactions in children. Ann N Y Acad Sci. 2007;1110:177-192.

42. International Conference on Harmonisation (ICH). ICH Guideline Q2(R1): Validation of Analytical Procedures: Text and Methodology. Geneva, Switzerland: ICH; 2008.

43. Uesugi M, Masuda S, Katsura T, Oike F, Takada Y, Inui K. Effect of intestinal CYP3A5 on postoperative tacrolimus trough levels in living-donor liver transplant recipients. Pharmacogenet Genomics. 2006;16:119-127.

44. Fanta S, Niemi M, Jönsson S, et al. Pharmacogenetics of cyclosporine in children suggests an age-dependent influence of ABCB1 polymorphisms. Pharmacogenet Genomics. 2008;18:77-90.

45. Herrlinger KR, Cummings JR, Barnardo MC, Schwab M, Ahmad T, Jewell DP. The pharmacogenetics of methotrexate in inflammatory bowel disease. Pharmacogenet Genomics. 2005;15:705-711.

46. European Commission. Ethical Considerations for Clinical Trials Performed in Children-Recommendations of the Ad $82 \mathrm{Hoc}$ Group for the Development of Implementing Guidelines for Directive 2001/20/EC Relating to Good Clinical Practice in the Conduct of Clinical Trials on Medicinal Products for Human Use (draft). Brussels, Belgium: European Commission; 2008.

47. Kishi S, Cheng C, French D, et al. Ancestry and pharmacogenetics of antileukemic drug toxicity. Blood. 2007;109:4151-4157.

48. Klotsman M, York TP, Pillai SG, et al. Pharmacogenetics of the 5-lipoxygenase biosynthetic pathway and variable clinical response to montelukast. Pharmacogenet Genomics. 2007;17:189-196.

49. Girnita DM, Webber SA, Ferrell R, et al. Disparate distribution of 16 candidate single nucleotide polymorphisms among racial and ethnic groups of pediatric heart transplant patients. Transplantation. 2006;82:1774-1780.
50. Frost BM, Forestier E, Gustafsson G, et al. Translocation $t(1 ; 19)$ is related to low cellular drug resistance in childhood acute lymphoblastic leukaemia. Leukemia. 2005;19:165-169.

51. Hawkins GA, Tantisira K, Meyers DA, et al. Sequence, haplotype, and association analysis of ADRbeta2 in a multiethnic asthma case-control study. Am J Respir Crit Care Med. 2006;174: 1101-1109.

52. Tsai HJ, Shaikh N, Kho JY, et al. Beta 2-adrenergic receptor polymorphisms: pharmacogenetic response to bronchodilator among African American asthmatics. Hum Genet. 2006;119:547-557.

53. Joober R, Grizenko N, Sengupta S, et al. Dopamine transporter 3'-UTR VNTR genotype and ADHD: a pharmaco-behavioural genetic study with methylphenidate. Neuropsychopharmacology. 2007;32:1370-1376.

54. Gilbert DL, Wang Z, Sallee FR, et al. Dopamine transporter genotype influences the physiological response to medication in ADHD. Brain 2006;129:2038-2046.

55. Sun HY, Hung CC, Lin PH, et al. Incidence of abacavir hypersensitivity and its relationship with HLA-B*5701 in HIV-infected patients in Taiwan. J Antimicrob Chemother. 2007;60:599-604.

56. Schaaf HS, Parkin DP, Seifart HI, et al. Isoniazid pharmacokinetics in children treated for respiratory tuberculosis. Arch Dis Child. 2005;90:614-618.

57. Lammer EJ, Shaw GM, Iovannisci DM, Van Waes J, Finnell RH. Maternal smoking and the risk of orofacial clefts: susceptibility with NAT1 and NAT2 polymorphisms. Epidemiology. 2004;15: 150-156.

58. Dos SC, Essioux L, Teinturier C, Tauber M, Goffin V, Bougneres P. A common polymorphism of the growth hormone receptor is associated with increased responsiveness to growth hormone. Nat Genet. 2004;36:720-724.

59. Sanderson S, Emery J, Higgins J. CYP2C9 gene variants, drug dose, and bleeding risk in warfarin-treated patients: a HuGEnet systematic review and meta-analysis. Genet Med. 2005;7:97-104.

60. Larramendy-Gozalo C, Yang JQ, Verstuyft C, et al. Genetic polymorphism of vitamin K epoxide reductase (VKORC1) 1173C $>\mathrm{T}$ in a Chinese and a Caucasian population. Basic Clin Pharmacol Toxicol. 2006;98:611-613.

61. Kaniwa N, Saito Y, Aihara M, et al. HLA-B*1511 is a risk factor for carbamazepine-induced Stevens-Johnson syndrome and toxic epidermal necrolysis in Japanese patients. Epilepsia. 2010;51: 2461-2465.

62. Man CB, Kwan P, Baum L, Yu E, Lau KM, Cheng AS, Ng MH. Association between HLA-B*1502 allele and antiepileptic druginduced cutaneous reactions in Han Chinese [Erratum in: Epilepsia. 2008;49:941]. Epilepsia. 2007;48:1015-1018.

63. Tassaneeyakul W, Tiamkao S, Jantararoungtong T, et al. Association between HLA-B ${ }^{*} 1502$ and carbamazepine-induced severe cutaneous adverse drug reactions in a Thai population. Epilepsia. 2010;51:926-930.

64. Mehta TY, Prajapati LM, Mittal B, et al. Association of HLA$\mathrm{B}^{*} 1502$ allele and carbamazepine- induced Stevens-Johnson syndrome among Indians. Indian J Dermatol Venereol Leprol. 2009;75:579-582.

For reprints and permission queries, please visit SAGE's Web site at http://www.sagepub.com/journalsPermissions.nav. 